\title{
Osteonecrose da cabeça do úmero: Avaliação dos resultados da artroplastia parcial com seguimento mínimo de 10 anos*
}

\section{Humeral Head Osteonecrosis: Outcomes of Hemiarthroplasty After Minimum 10-Year Follow-Up}

\author{
Alberto Naoki Miyazaki ${ }^{1}$ Guilherme do Val Sella ${ }^{2}$ Luciana Andrade da Silva ${ }^{2}$ Caio Santos Checchia ${ }^{2}$ \\ Felipe Cerávolo Lemos ${ }^{2}$ \\ ${ }^{1}$ Departamento de Ortopedia e Traumatologia, Faculdade de Ciências \\ Médicas, Grupo de Cirurgia de Ombro e Cotovelo, Santa Casa de \\ São Paulo, São Paulo, SP, Brasil \\ ${ }^{2}$ Grupo de Cirurgia de Ombro e Cotovelo, Santa Casa de São Paulo, \\ São Paulo, SP, Brasil \\ Rev Bras Ortop 2021;56(1):91-97. \\ Endereço para correspondência Luciana Andrade da Silva, Rua \\ Estuário, 519, Chacara Monte Alegre, São Paulo, SP, 04645-100, Brasil \\ (e-mail: lucalu@terra.com.br).
}

\section{Resumo}

Palavras-chave

- osteonecrose

- cabeça do úmero

- seguimentos
Objetivo Analisar os resultados funcionais e radiográficos de longo prazo da artroplastia parcial do ombro para estosteonecrose da cabeça do úmero.

Métodos Revisão retrospectiva de 13 casos, com seguimento pós-operatório médio de 17 anos (variação de 10 a 26 anos). Os achados do último seguimento foram comparados àqueles em que os pacientes tinham com 1 ano de acompanhamento pósoperatório. A avaliação funcional consistiu em medidas do movimento do ombro e aplicação do escore do ombro da Universidade da Califórnia, Los Angeles (UCLA). Todos os pacientes foram submetidos a exame radiografico para medir a erosão glenoidal, a migração umeral proximal, e o deslocamento glenoumeral lateral.

Resultados A erosão da glenoide aumentou com o tempo significativamente $(p<0,05)$. Paradoxalmente, todos os movimentos ativos do ombro também melhoraram $(p<0,05)$, enquanto os escores da UCLA permaneceram os mesmos. A deterioração radiográfica não teve correlação com a função clínica. Tivemos uma taxa de sobrevida de $84,7 \%$ das artroplastias após tempo médio de 16 anos.

Conclusões Os resultados funcionais precoces mantiveram-se a longo prazo e não se correlacionem com a deterioração radiográfica (aumento da erosão glenoidal).

Trabalho realizado no Grupo de Cirurgia de Ombro e Cotovelo do Departamento de Ortopedia e Traumatologia da Faculdade de Ciências Médicas da Santa Casa de São Paulo, "Pavilhão Fernandinho Simonsen" (DOT - FCMSCSP) (Diretor: Professor Doutor Ivan Chakkour).

recebido

21 de Junho de 2019

aceito

03 de Outubro de 2019

Publicado online

Março 16, 2020
DOI https://doi.org/

10.1055/s-0039-3402471. ISSN 0102-3616. (c) 2020. Sociedade Brasileira de Ortopedia e Traumatologia. All rights reserved.

This is an open access article published by Thieme under the terms of the Creative Commons Attribution-NonDerivative-NonCommercial-License, permitting copying and reproduction so long as the original work is given appropriate credit. Contents may not be used for commercial purposes, or adapted, remixed, transformed or built upon. (https://creativecommons.org/ licenses/by-nc-nd/4.0/)

Thieme Revinter Publicações Ltda., Rua do Matoso 170, Rio de Janeiro, RJ, CEP 20270-135, Brazil 


\begin{abstract}
Objective To analyze long-term functional and radiographic results of partial shoulder replacement for humeral head osteonecrosis.

Methods Retrospective review of thirteen cases, with a mean postoperative followup of 17 years (range 10 to 26 years). The findings from the last follow-up were compared to those in which the patients had one year of postoperative follow-up. Functional assessment consisted of shoulder movement measurements and application of the University of California, Los Angeles (UCLA) shoulder score. All patients underwent radiographic examination to measure glenoid erosion, proximal humeral migration and lateral glenohumeral dislocation.

Results Glenoid erosion increased over time significantly $(p<0.05)$. Paradoxically, all active shoulder movements also improved $(p<0.05)$, while UCLA scores remained the

Keywords

- osteonecrosis

- humeral head

- follow-up studies same. Radiographic deterioration was not correlated with clinical function. We had an $84.7 \%$ survival rate for arthroplasties after a mean time of 16 years.

Conclusions Early functional outcomes were maintained in the long run and do not correlate with radiographic deterioration (increased erosion of the glenoid).
\end{abstract}

\section{Introdução}

A osteonecrose da cabeça do úmero é uma afecção rara, mas uma causa significante de dor na articulação do ombro que, em muitos casos, responde mal ao tratamento não cirúrgico. ${ }^{1-3}$ Ela corresponde a aproximadamente $5 \%$ do diagnóstico pré-operatório de todas as artroplastias de ombro realizadas. ${ }^{4}$

Quando opta-se pelo tratamento cirúrgico da osteonecrose, dependendo do grau de acometimento da superfície articular, a indicação mais comum é artroplastia. ${ }^{2,5,6} \mathrm{~A}$ decisão quanto à escolha entre a artroplastia parcial e a total é, em geral, baseada no estado da cartilagem da cavidade glenoidal durante o ato operatório. Neer classificou a doença em 4 estágios: no estágio 3 de Neer, há colapso do osso subcondral de uma área da cabeça do úmero. A cartilagem nessa região apresenta-se irregular e pode soltar-se. Neste estágio é preconizado o uso da artroplastia parcial do ombro. Já no estágio 4 , onde há envolvimento também da superfície articular da cavidade glenoidal, geralmente é indicada a artroplastia total. No entanto, se houver uma lesão dos tendões do manguito rotador, uma artrose concêntrica e/ou se o cirurgião julgar que o estoque ósseo da cavidade glenoidal é muito ruim, existe sempre a opção de realizar uma artroplastia parcial ${ }^{6}$ (-Fig. 1).
A literatura mostra que o uso da artroplastia parcial para o tratamento cirúrgico da osteonecrose é eficaz para o alívio da dor, para aumento da mobilidade do ombro e do grau de satisfação dos pacientes, mesmo quando comparada aos resultados da artroplastia total. $2,4,6,7$

Pollock et al., ${ }^{8}$ em 1996, em um estudo de avaliação clínica, obteve bons resultados com o uso da artroplastia parcial nas artroses concêntricas (quando a cabeça umeral permanece centralizada com a cavidade glenoidal), assim como na artroplastia total nas artroses excêntricas (onde existe uma incongruência da cabeça do úmero que leva a um desgaste desigual da glenóide e eventualmente a cabeça do úmero subluxa posterior). (-Fig. 2) Em 2001, nosso grupo avaliou 21 pacientes com osteonecrose da cabeça do úmero que foram submetidos a artroplastias (totais e parciais), realizadas no serviço, com seguimento médio de 37 meses. Concluiu-se nesse estudo que a artroplastia, total ou parcial, é um bom procedimento para o alívio da dor e recuperação da função articular desses pacientes. ${ }^{9}$

No entanto, poucos estudos compararam os resultados a longo prazo das artroplastias totais com as hemiartroplastias nos casos de osteonecrose. Gadea et al., ${ }^{10}$ em 2012, mostraram uma taxa de "sobrevivência" da artroplastia de $94 \%$

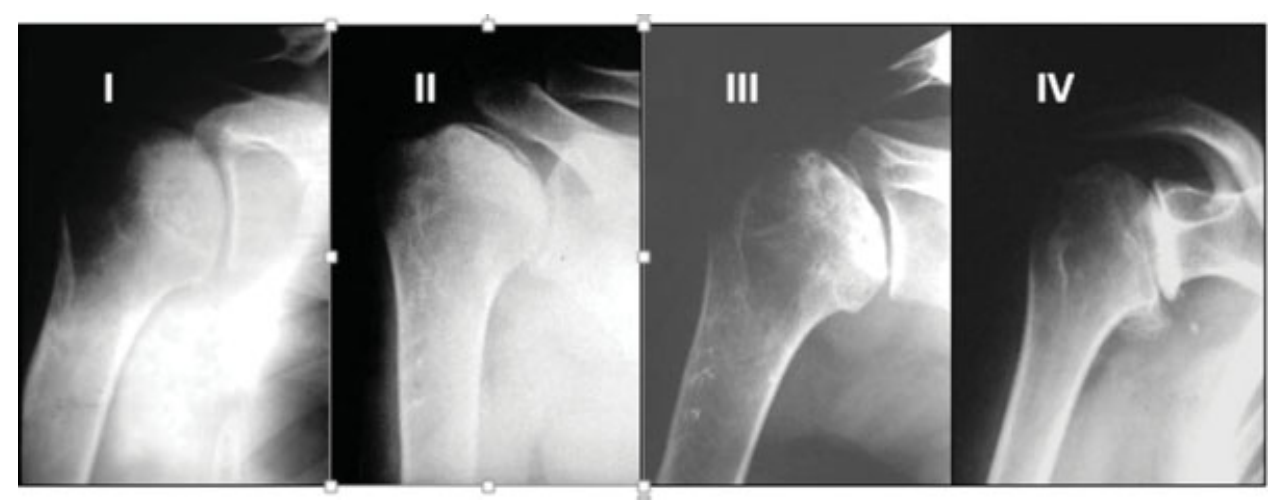

Fig. 1 Aspecto radiográfico das diferentes fases da osteonecrose da cabeça do úmero, segundo a classificação de Neer. ${ }^{6}$ 


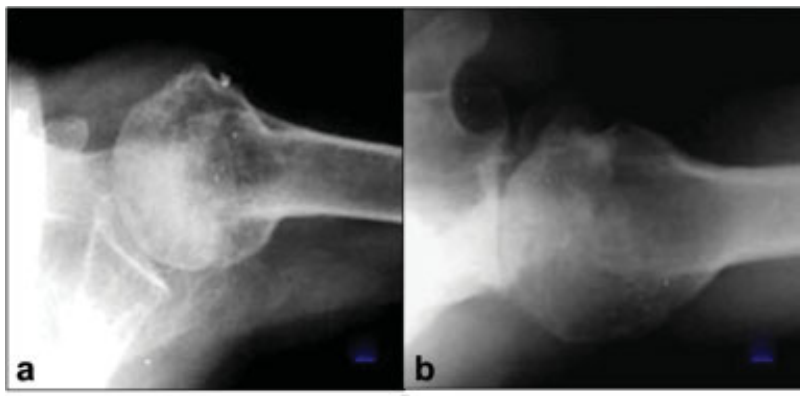

Fig. 2 Osteonecrose da cabeça do úmero tipo concêntrica (a) e excêntrica (b), segundo Pollock et al. ${ }^{8}$

considerando um seguimento de 10 anos. Os autores concluíram que a artroplastia parcial do ombro é uma indicação confiável na osteonecrose da cabeça do úmero, independente da etiologia. Entretanto, no mesmo estudo, os autores chamam atenção para o fato de que apesar dos resultados funcionais semelhantes, observou-se que a taxa de "sobrevivência" da prótese total foi maior. ${ }^{10}$

O desenvolvimento de erosão da cavidade glenoidal é uma complicação conhecida da artroplastia parcial do ombro, sendo a principal causa de evolução desfavorável a médio e longo prazo, resultando em dor, perda progressiva da amplitude de movimentos, diminuição da função do ombro e, conseqüentemente, aumento dos índices de insatisfação do paciente. ${ }^{4,11-14}$ Herschel et al. ${ }^{11}$ mostraram que a cavidade glenoidal desenvolveu algum grau de erosão visível ao exame radiográfico em $89 \%$ dos pacientes operados após um seguimento médio de 51 meses.

O objetivo desse estudo é verificar se houve a manutenção dos resultados funcionais dos nossos pacientes que foram submetidos a artroplastia parcial devido a osteonecrose ao longo de 10 anos, se eles tiveram progressão da erosão e se seus resultados clínicos têm correlação com os aspectos radiográficos encontrados nos exames atuais.

\section{Casuística e Métodos}

Entre dezembro de 1988 e fevereiro de 2008, foram realizadas 27 artroplastias parciais em 27 ombros de 25 pacientes para o tratamento da osteonecrose.

Foram incluídos em nosso estudo todos os pacientes com osteonecrose da cabeça do úmero, independente da etiologia ou grau de acometimento, que foram submetidos a artroplastia parcial e que completaram um tempo de seguimento mínimo de 10 anos de pós-operatório. Os pacientes que não preencheram os critérios acima foram excluídos do estudo.

Dos 27 ombros conseguimos reavaliar 13, com seguimento variando de 10 a 26 anos (média de 16,8 anos). Doze pacientes ( 14 ombros, pois 2 possuíam artroplastia parcial bilateral) foram excluídos: 5 porque não foram localizados e 7 porque faleceram, não completando um seguimento de 10 anos (-Tabela 1).

A idade dos pacientes na última avaliação variou de 42 a 92 anos (média de 71 anos). Seis $(46,15 \%)$ pacientes eram do sexo masculino e 7 (53,85\%) do sexo feminino. Em 8 (61,54\%) situações, o lado dominante foi o acometido.

Com relação à etiologia, tivemos 8 (61\%) ombros com necrose pós-traumática, 3 (23\%) ombros com necrose idiopática, 1 ombro (7,7\%) devido a anemia falciforme e 1 ombro $(7,7 \%)$ devido a disbarismo.

O grau de acometimento da articulação foi avaliado pelo estadiamento de Ficat e Enneking, ${ }^{15}$ modificado por Neer, ${ }^{6}$ sendo $10(77 \%)$ casos de necrose no estágio III e 3 (23\%) no estágio IV. (-Fig. 1) Todos os casos no estágio IV foram considerados como artrose do tipo concêntrica. ${ }^{8}$

Todos os pacientes foram submetidos a artroplastia parcial por via de acesso deltopeitoral. Todas as artroplastias tiveram a haste umeral cimentada, e os modelos usados

Tabela 1 Dados clínicos dos pacientes

\begin{tabular}{|l|l|l|l|l|l|l|l|}
\hline Caso & Idade (a) & Sexo & Dom & Etiologia & Neer (estágio) & Tipo de Prótese & Seguimento (a) \\
\hline 1 & 59 & F & - & Pós-trauma & III & Neer II & 27 \\
\hline 2 & 73 & F & - & Idiopática & III & Neer II & 24 \\
\hline 3 & 74 & M & + & Disbarismo & III & Neer II & 23 \\
\hline 4 & 57 & M & - & Idiopática & III & Eccentra & 20 \\
\hline 5 & 92 & F & + & Pós-trauma & IV & Neer II & 19 \\
\hline 6 & 68 & M & + & Pós-trauma & III & Eccentra & 18 \\
\hline 7 & 74 & M & - & Pós-trauma & III & Neer II & 15 \\
\hline 8 & 62 & M & + & Pós-trauma & III & Eccentra & 13 \\
\hline 9 & 72 & M & - & Pós-trauma & III & Eccentra & 13 \\
\hline 10 & 79 & F & + & Pós-trauma & IV & Neer II & 12 \\
\hline 11 & 42 & F & + & Anemia falciforme & III & Eccentra & 12 \\
\hline 12 & 88 & F & + & Idiopática & IV & Neer II & 10 \\
\hline 13 & 85 & F & + & Pós-trauma & III & Eccentra & 10 \\
\hline
\end{tabular}

Fonte: Arquivos médicos da instituição.

Legenda: a, ano, DOM, dominância; F, feminino; M, masculino. 
foram, em 7 casos, o modelo Neer II (Memphis, Tennessee, US), e em 6, o modelo Eccentra (São Paulo, SP, Brasil).

No pós-operatório, o paciente é imobilizado por 6 semanas em tipoia tipo "Velpeau". Iniciado fisioterapia com exercícios pendulares e de rotação externa até neutra, passivamente, desde o $1^{\circ}$ dia pós operatório. Os movimentos ativos são introduzidos a partir da $6^{\text {a }}$ semana.

Para medirmos o grau de mobilidade da articulação, utilizamos o método da Academia Americana dos Cirurgiões Ortopedistas (AAOS, na sigla em inglês). ${ }^{16}$ Para a avaliação funcional dos pacientes, utilizamos o método da University of California, Los Angeles (UCLA). ${ }^{17}$

Todos os pacientes foram submetidos a reavaliação por imagens. Foram realizadas radiografias do ombro nas posições frente corrigida, axilar, e túnel do supra-espinal. Na radiografia de frente corrigida, medimos alguns parâmetros baseados no método publicado por por Ohl et al. ${ }^{18}$ :

- offset glenoumeral lateral: definido como a distância entre a margem lateral do tubérculo maior e a base do processo coracóide;

- espaço articular glenoumeral: definido como a região onde o espaço articular se encontra mais estreito entre a prótese e a glenoide;

- profundidade da cavidade glenoidal: mensurada entre o centro da glenóide e uma linha passando entre o ápice e a margem inferior da glenoide;

- migração proximal do úmero, definida como a distância entre uma linha horizontal passando pela margem inferior da glenoide e uma linha horizontal passando pelo implante da cabeça umeral;

- espaço subacromial, determinado pela distância que separa o limite superior do tubérculo maior e a margem inferior do acrômio (-Fig. 3).

O grau de erosão da cavidade glenoidal foi avaliada na radiografia do ombro, incidência de frente corrigida, através do método proposto por Sperling et al. ${ }^{19}$ e Herschel et al. ${ }^{11}$ em 2007, sendo graduada em:

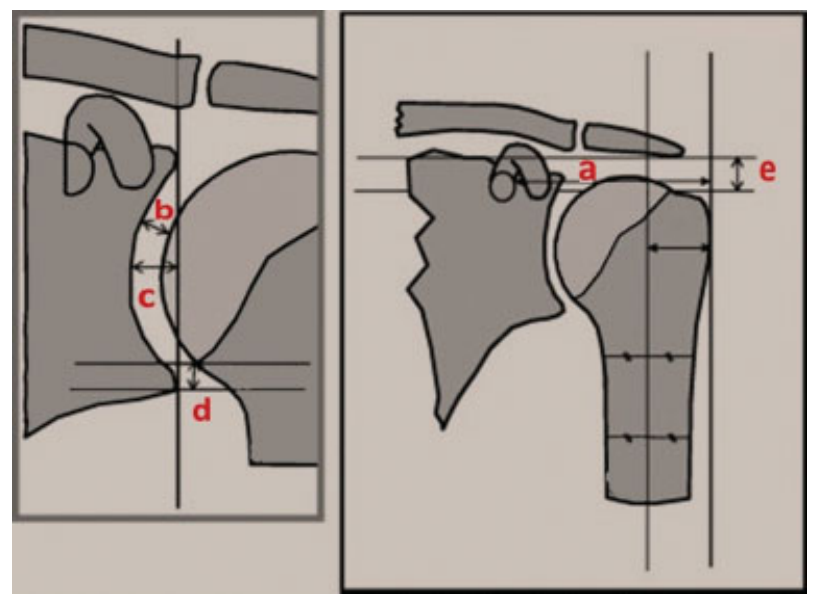

Fig. 3 llustração mostrando os diferentes parâmetros radiográficos utilizados: (a) offset glenoumeral lateral, (b) espaço articular, (c) profundidade da cavidade glenoidal (CG), (d) migração proximal (Mp), (e) espaço subacromial.

\section{- Ausente (grau I);}

- Leve (grau II), erosão no osso subcondral;

- Moderada (grau III), medialização do osso subcondral com deformação hemisférica da glenoide;

- Grave (grau IV), completa deformação hemisférica da glenoide com perda óssea superior à base do processo coracoide.

Para a amostra de 13 pacientes, foram inicialmente calculadas as distribuições de frequência, alguns testes estatísticos descritivos e construídos gráficos de boxplot para ilustração da comparação entre as situações iniciais (PO1a) e de seguimento (PO10a), bem como para identificação de possíveis observações discrepantes.

Como a amostra foi considerada homogênea, possibilitou o uso do teste $\mathrm{t}$ de Student e o teste não paramétrico de Mann-Whitney. ${ }^{20}$

Foi adotado um nível de significância de 5\%, sendo rejeitadas as hipóteses com níveis descritivos (valores- $p$ ) inferiores a este valor. As análises foram realizadas utilizando-se o programa estatístico Minitab®, v.17 (Minitab LLC., State College, PA, EUA). ${ }^{20}$

O protocolo do estudo foi aprovado pelo comitê de ética da instituição.

\section{Resultados}

Após avaliação dos 13 pacientes operados, comparamos a amplitude de movimento com um ano de pós-operatório (PO1a) com a encontrada na avaliação após 10 anos (PO10a). Os valores estão ilustrados na - Tabela 2, que nos mostra que houve ganho em todas as direções do movimento, com aumento médio de $5^{\circ}$ de elevação, $2^{\circ}$ de rotação lateral e 2 níveis vertebrais mediais. Os valores obtidos apresentaram nível de significância de $5 \%$.

A média de escala da UCLA ${ }^{17}$ no PO1a foi de 24 (17-30) e no P010a foi de 24,5 pontos (variou de 14 a 34), mostrando que o UCLA ${ }^{17}$ não se modificou após 10 anos de seguimento pós-operatório.

No seguimento dos pacientes P01a, tivemos 4 bons resultados (30,8\%), 6 razoáveis ou regulared $(46,2 \%)$, e $3(23,1 \%)$ pacientes com resultado ruim. Se compararmos com os resultados dos pacientes $\mathrm{PO} 10 \mathrm{a}$, foram encontrados resultados excelentes em 1 caso (7,7\%), bons em 5 (38,5\%), razoável ou regular em 3 (23,1\%), e ruins em 4 pacientes (30,8\%). Em relação ao grau de satisfação, $4(30,8 \%)$ PO1a pacientes

Tabela 2 Mobilidade articular média dos pacientes

\begin{tabular}{|l|l|l|l|}
\hline Média & PO 1 a & PO 10 a & Valor-p \\
\hline Elevação & $100^{\circ}$ & $105^{\circ}$ & $p>0,100$ \\
\hline Rotação externa & $34^{\circ}$ & $36^{\circ}$ & $p>0,100$ \\
\hline Rotação interna & L3 & L1 & $p>0,100$ \\
\hline
\end{tabular}

Fonte: Arquivos médicos da instituição.

Legenda: a, anos; PO, pós operatório. 
Tabela 3 Mensuração radiográfica do grau de erosão da cavidade glenoidal após 1 e 10 anos de cirurgia, avaliada pelo método proposto por Sperling et al. ${ }^{19}$

\begin{tabular}{|l|l|l|}
\hline Grau & $\begin{array}{l}\text { PO 1 a } \\
\mathbf{n}^{\circ}(\%)\end{array}$ & $\begin{array}{l}\text { PO 10 a } \\
\mathbf{n}^{\circ}(\%)\end{array}$ \\
\hline I & $01(08)$ & - \\
\hline II & $10(77)$ & $01(08)$ \\
\hline III & $01(08)$ & $09(69)$ \\
\hline IV & $01(08)$ & $03(23)$ \\
\hline
\end{tabular}

Fonte: Arquivos médicos da instituição.

Legenda: a, anos; $\mathrm{n}^{\circ}$, número; PO, pós-operatório.

Tabela 4 Avaliação dos parâmetros radiográficos do ombro encontrados através do método proposto por Ohl et al. ${ }^{18}$

\begin{tabular}{|l|l|l|l|}
\hline & $\begin{array}{l}\text { PO 1 a } \\
(\mathrm{mm})\end{array}$ & $\begin{array}{l}\text { PO 10 a } \\
(\mathrm{mm})\end{array}$ & $\begin{array}{l}\text { PO 1 a-PO } \\
10 \mathrm{a}(\mathrm{mm})\end{array}$ \\
\hline $\begin{array}{l}\text { Off-set glenoumeral } \\
\text { lateral }\end{array}$ & 67,3 & 63,6 & 3,7 \\
\hline $\begin{array}{l}\text { Espaço articular } \\
\text { glenoumeral }\end{array}$ & 2,07 & 1,15 & 0,92 \\
\hline $\begin{array}{l}\text { Profundida da } \\
\text { cavidade glenoidal }\end{array}$ & 4,1 & 8,53 & $-4,38$ \\
\hline Migração proximal & 4,42 & 6,31 & $-1,88$ \\
\hline Espaço subacromial & 8,07 & 5,38 & 2,69 \\
\hline
\end{tabular}

Fonte: Arquivos médicos da instituição.

Legenda: PO 1 a, valores encontrados com um ano de pós-operatório; PO 10 a, valores encontrados no seguimento após tempo mínimo de 10 anos; PO 1 a-PO 10 a, diferença entre ambos.

encontravam-se insatisfeitos com , ao passo que apenas 2 $(15,4 \%)$ P010a pacientes encontravam-seinsatisfeitos.

Em relação à avaliação radiográfica do grau de erosão avaliada pelo método proposto por Sperling et al. ${ }^{19}$ e Herschel et al., ${ }^{11}$ houve aumento médio do desgaste da cavidade glenoidal de um estágio durante o seguimento, isto é, em média no P01a, os pacientes tinham uma erosão classificada como estágio II e agora no PO10a, a erosão encontrada foi classificada como estágio III. A avaliação completa está na - Tabela 3.

Na radiografia de frente corrigido, através do método proposto por Ohl et al., ${ }^{18}$ obtivemos os seguintes resultados: diminuição do offset glenoumeral lateral, do espaço articular glenoumeral e do espaço subacromial. Houve aumento da profundidade da cavidade glenoumeral e da migração proximal da cabeça do úmero, ilustrados na - Tabela 4. Todos com significância estatística $(p>0,1)$.

\section{Discussão}

Com o seguimento mínimo de 10 anos, obtivemos um aumento da mobilidade do ombro em todas as direções e uma média de UCLA ${ }^{17}$ de 24,5 (regular), isto é, ganho de meio ponto em relação a avaliação com um ano de pós-operatório.
Ao analisarmos isoladamente o aumento da profundidade da cavidade glenoidal (de 4,3 mm em média) e a diminuição do espaço articular glenoumeral (média de $0,9 \mathrm{~mm}$ ) que foram as alterações radiográficas mais evidentes, observamos que não houve correlação diretamente proporcional ao resultado do UCLA, ${ }^{17}$ que manteve-se ao redor de 24 .

De acordo com Gadea et al. ${ }^{10}$ e Smith et al., ${ }^{21}$ é reconhecido que a artroplastia parcial pode resultar em erosão da cavidade glenoidal, sendo esta a principal causa de deterioração clínica e de revisão para artroplastia total. Em um estudo realizado por Herschel et al., ${ }^{11} 89 \%$ dos pacientes desenvolveram algum grau de erosão da cavidade glenoidal visível ao exame radiográfico em um tempo médio de seguimento de 31 meses, porém apenas 10\% tiveram indicação de revisão cirúrgica. 0 mesmo pode-se observar no estudo realizado por Sperling et al., ${ }^{19}$ no qual com 7 anos de tempo médio de acompanhamento, apenas $24 \%$ dos pacientes foram submetidos a uma reoperação, sendo a erosão da cavidade glenoidal a causa mais comum da cirurgia (90\%).

Em nosso estudo, verificamos que todos os pacientes tiveram algum grau de erosão visível ao exame radiográfico após o seguimento mínimo de 10 anos de pós-operatório. Apesar da evolução radiográfica encontrada, obtivemos resultados funcionais satisfatórios, com uma taxa de sobrevida de $84,7 \%$ das artroplastias após tempo médio de 16 anos. Cerciello et al. ${ }^{22}$ encontraram uma incidência de $72 \%$ de erosão da cavidade glenoidal, associado a sintomatologia em 6 to $72 \%$ dos casos, o que apoia os resultados encontrados em nosso trabalho, de que nem sempre a erosão está associada a sintomas, e quando existem, podem não ser incapacitantes (-Fig. 4).

Quando perguntados sobre satisfação em relação ao resultado cirúrgico, 11 (84,7\%) pacientes encontravam-se satisfeitos com o resultado na última avaliação (PO10a), apesar do grau de alteração encontrados nos exames radiográficos. Acreditamos que isso se deva a uma adaptação clínica e funcional dos pacientes ao realizar suas atividades diárias, e por isso, apesar da deterioração radiográfica, os pacientes não se sentem mais incapacitados que anteriormente. E como satisfação é um dos parâmetros avaliados no critério do UCLA, ${ }^{17}$ isto afeta diretamente seu resultado.

Apenas 2 (15,4\%) pacientes manifestaram-se realmente insatisfeitos com seus resultados. Estes 2 apresentavam ao exame radiográfico no de $\mathrm{PO} 10 \mathrm{a}$ uma erosão grave da cavidade glenoidal classificada como grau IV de Sperling et al. $^{19}$

No primeiro caso, a paciente apresentou osteonecrose de causa idiopática, acometendo o membro dominante. Ela apresentava osteonecrose grau IV e artrose concêntrica na avaliação pré-operatória. Foi feita uma artroplastia parcial tipo Neer. Aos PO10a, a paciente apresentava 15 pontos na escala UCLA, ${ }^{17}$ com amplitude de movimento de $80^{\circ}, 20^{\circ}$, glúteo com dor e incapacidade funcional. Foi indicado o procedimento de revisão da hemiartroplastia para artroplastia total. Atualmente, encontra-se no $5^{\circ}$ ano pós-revisão, com dor controlada, e amplitude de movimento de $80^{\circ}, 40^{\circ}$, sacro (ganho de $20^{\circ}$ de rotação lateral). 


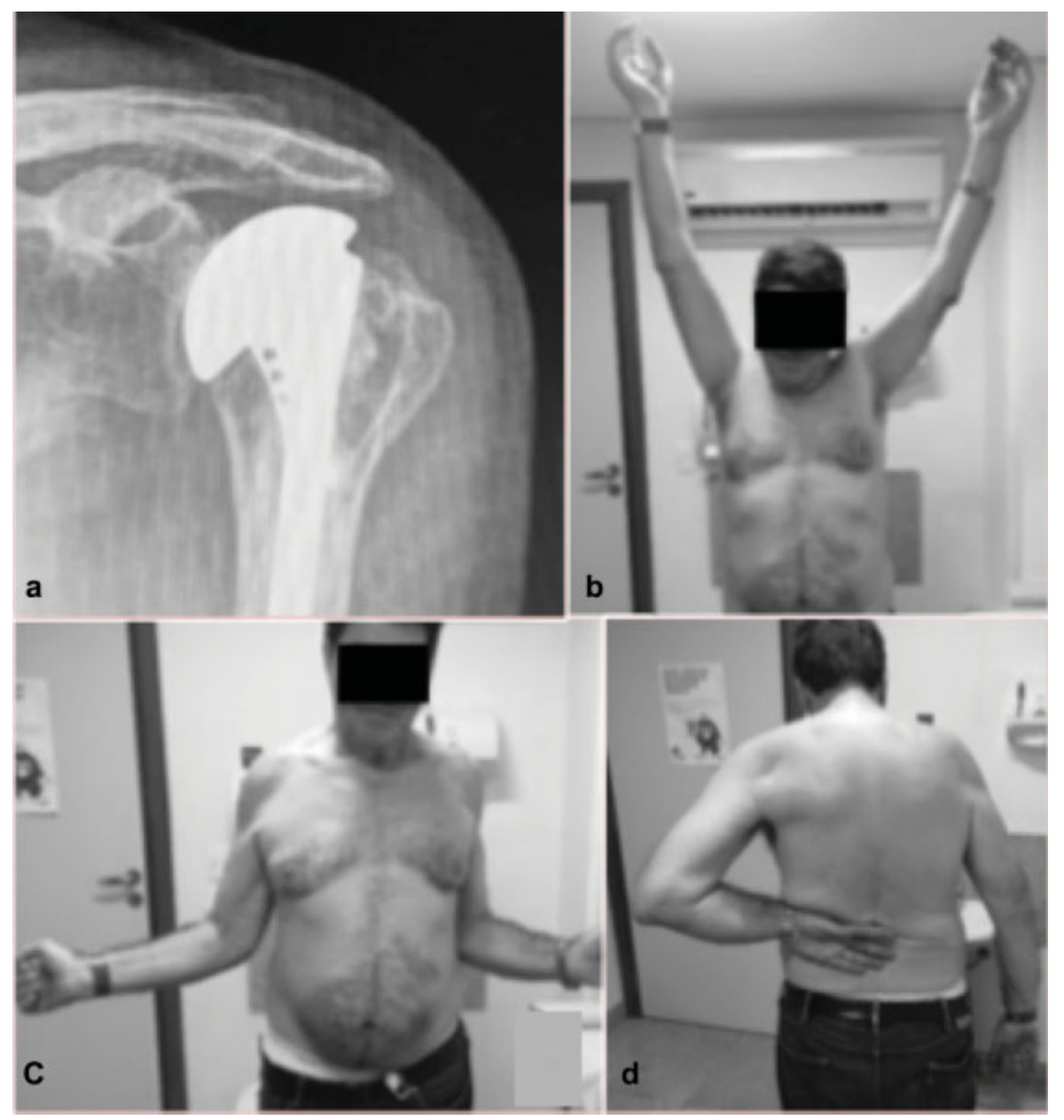

Fig. 4 Paciente aos 13 anos pós-cirurgia de artroplastia parcial por osteonecrose pós-trauma. (a) no exame radiográfico apresenta diminuição do espaço articular glenoumeral e erosão da cavidade glenoidal. Paciente com 34 pontos na classificação da Universidade da Califórnia, Los Angeles (UCLA), com boa mobilidade articular: (b) elevação, (c) rotação lateral e (d) medial.

Já o outro caso, é a paciente mais jovem da nossa casuística (45 anos), sendo a etiologia da necrose secundária a anemia falciforme. A paciente possui 12 anos de pós-operatório, com escore de 14 pontos na escala da UCLA, ${ }^{17}$ amplitude de movimento de $90^{\circ}, 0^{\circ}$, L4. Com 1 ano de pósoperatório, a paciente apresentava um UCLA ${ }^{17}$ de 24 , com $80^{\circ}, 20^{\circ}$, glúteo. 0 membro operado é o membro dominante, porém no membro contra-lateral a paciente também tem osteonecrose da cabeça do úmero, classificada como grau II, fato que está dificultando muito a realização das atividades de vida diária. Portanto, apesar da pequena perda de amplitude de movimento durante o seguimento acreditamos que o acometimento contra-lateral esteja contribuindo para a sua insatisfação e dificuldade de adaptação. A paciente encontrase em acompanhamento ambulatorial no grupo com indicação de tratamento cirúrgico do lado contra-lateral, porém devido a problemas particulares não quer fazer a cirurgia no momento.

Dos 7 pacientes que apresentaram resultado insatisfatório no P010a, pudemos observar que foi utilizada prótese tipo Neer II em 5; no entanto, do ponto de vista estatístico ( $p>5 \%)$ não houve diferença quando do uso da prótese Eccentra e
Neer II em relação ao UCLA ${ }^{17}$ e ao arco de movimento entre os seguimentos de PO1 e PO10 anos.

\section{Conclusão}

O estudo nos mostra que houve a manutenção dos resultados funcionais dos nossos pacientes que foram submetidos a artroplastia parcial devido a osteonecrose da cabeça do úmero ao longo de 10 anos. Foi observada progressão da erosão da cavidade glenoidal, mas os resultados clínicos, em $85 \%$ dos pacientes, não apresentaram correlação com a deterioração dos aspectos radiográficos encontrados nos exames atuais.

\section{Conflito de Interesses}

Os autores declaram não haver conflito de interesses.

\section{Referências}

1 Loebenberg MI, Plate AM, Zuckerman JD. Osteonecrosis of the humeral head. Instr Course Lect 1999;48:349-357

2 Feeley BT, Fealy S, Dines DM, Warren RF, Craig EV. Hemiarthroplasty and total shoulder arthroplasty for avascular necrosis of the humeral head. J Shoulder Elbow Surg 2008;17(05):689-694 
3 Hernigou P, Flouzat-Lachaniette $\mathrm{CH}$, Roussignol X, Poignard A. The natural progression of shoulder osteonecrosis related to corticosteroid treatment. Clin Orthop Relat Res 2010;468(07):1809-1816

4 Schoch BS, Barlow JD, Schleck C, Cofield RH, Sperling JW. Shoulder arthroplasty for post-traumatic osteonecrosis of the humeral head. J Shoulder Elbow Surg 2016;25(03):406-412

5 Parsch D, Lehner B, Loew M. Shoulder arthroplasty in nontraumatic osteonecrosis of the humeral head. J Shoulder Elbow Surg 2003;12(03):226-230

6 Neer CS. Glenohumeral arthroplasty. In: Shoulder reconstruction. Philadelphia: W.B. Saunders; 1990:143-227

7 Orfaly RM, Rockwood CA Jr, Esenyel CZ, Wirth MA. Shoulder arthroplasty in cases with avascular necrosis of the humeral head. J Shoulder Elbow Surg 2007;16(3, Suppl)S27-S32

8 Pollock R, Glasson JM, Djurasovic M, et al. Hemiarthroplasty for glenohumeral osteoarthrits in pacients with an intact rotador cuff: results correlated to degree of glenoid wear. In: AAOS Twelvth Open Meeting, Atlanta GA, February 25, 1996.

9 Checchia SL, Santos PD, Miyazaki AN, et al. Osteonecrose da cabeça do úmero: resultados das artroplastias. Rev Bras Ortop 2001;36(12):29-36

10 Gadea F, Alami G, Pape G, Boileau P, Favard L. Shoulder hemiarthroplasty: outcomes and long-term survival analysis according to etiology. Orthop Traumatol Surg Res 2012;98(06):659-665

11 Herschel R, Wieser K, Morrey ME, Ramos CH, Gerber C, Meyer DC. Risk factors for glenoid erosion in patients with shoulder hemiarthroplasty: an analysis of 118 cases. J Shoulder Elbow Surg 2017;26(02):246-252

12 Schoch BS, Barlow JD, Schleck C, Cofield RH, Sperling JW. Shoulder arthroplasty for atraumatic osteonecrosis of the humeral head. J Shoulder Elbow Surg 2016;25(02):238-245

13 Mansat $\mathrm{P}$, Huser L, Mansat $\mathrm{M}$, Bellumore $\mathrm{Y}$, Rongières $\mathrm{M}$, Bonnevialle P. Shoulder arthroplasty for atraumatic avascular necrosis of the humeral head: nineteen shoulders followed up for a mean of seven years. J Shoulder Elbow Surg 2005;14(02): $114-120$

14 Cofield RH. Total shoulder arthroplasty with the Neer prosthesis. J Bone Joint Surg Am 1984;66(06):899-906

15 Satterlee CC. Osteonecrosis and other non-inflammatory degenerative diseases of the glenohumeral joint including Gaucher's diseases, sickle cell diseases, hemochromatosis and synovial osteochondromatosis. In: AAOS Instructional Course Lectures. RosemontIL1997:233-240

16 Hawkins JR, Bokor DJ. Clinical evaluation of shoulder problems. In: Rockwood CA, Matsen FA, editors. The shoulder. Philadelphia: Saunders; 1990:149-177

17 Ellman H, Kay SP. Arthroscopic subacromial decompression for chronic impingement. Two- to five-year results. J Bone Joint Surg Br 1991;73(03):395-398

18 Ohl X, Nérot C, Saddiki R, Dehoux E. Shoulder hemi arthroplasty radiological and clinical outcomes at more than two years followup. Orthop Traumatol Surg Res 2010;96(03):208-215

19 Sperling JW, Cofield RH, Schleck CD, Harmsen WS. Total shoulder arthroplasty versus hemiarthroplasty for rheumatoid arthritis of the shoulder: results of 303 consecutive cases. J Shoulder Elbow Surg 2007;16(06):683-690

20 Montgomery DC, Runger GC. Estatística aplicada e probabilidade para engenheiros. 6a. ed. Rio de Janeiro: LTC; 2016

21 Smith RG, Sperling JW, Cofield RH, Hattrup SJ, Schleck CD. Shoulder hemiarthroplasty for steroid-associated osteonecrosis. J Shoulder Elbow Surg 2008;17(05):685-688

22 Cerciello S, Morris BJ, Visonà E, et al. No relationship between critical shoulder angle and glenoid erosion after shoulder hemiarthroplasty: a comparative radiographic study. Arch Orthop Trauma Surg 2017;137(07):919-923 